\title{
Levcromakalim (BRL38227): A Potassium Channel Activator Alters Some Biochemical Parameters in Streptozotocin- Induced Diabetic Rats
}

\section{O J Owolabi* and E K I Omogbai}

Department of Pharmacology and Toxicology, Faculty of Pharmacy, University of Benin, Benin City, Edo State, Nigeria

\begin{abstract}
Levcromakalim, a potassium channel activator reduces blood pressure and hence used in the management of hypertension, known to co-exist with diabetes mellitus; hence its effect on creatinine, urea and 24 hour urine output was investigated in streptozotocin- induced diabetic rats.

The rats were given levcromakalim $(75 \mu \mathrm{g} / \mathrm{kg} /$ day $)$ for 4 consecutive weeks, thereafter experimental diabetes mellitus was induced using streptozotocin $(60 \mathrm{mg} / \mathrm{kg})$. The rats were randomly placed into groups of 5 each and transferred to metabolic cages after treatment with glibenclamide $(5 \mathrm{mg} / \mathrm{kg})$, metformin $(350 \mathrm{mg} / \mathrm{kg})$ and insulin $(5,10$, 20 and 40 i.u/kg).
\end{abstract}

The following parameters were investigated 24 hours after drug administration: plasma creatinine and urea, urine creatinine and urea, creatinine clearance and the $24 \mathrm{~h}$ urine volume.

The result points to a significant reduction $(p<0.05)$ in the $24 \mathrm{~h}$ urine volume on treatment with levcromakalim in diabetic rats in comparison with those diabetic not treated with levcromakalim.

A significant reduction $(p<0.05)$ of the plasma creatinine was also noted on treatment with levcromakalim in the diabetic rats, while an increase in urine creatinine was observed when compared with those not treated. Levcromakalim also significantly $(p<0.05)$ increased the creatinine clearance of normal rats, those of the diabetic rats were not significant.

In conclusion treatment with levcromakalim affords a renoprotective effect to diabetic and normal rats as evident from its effect on plasma creatinine and the creatinine clearance. Secondly it also corrects polyuria a known symptom of diabetes mellitus.

Keywords: Potassium activator; Creatinine; Reno-protection; Urine volume

\section{Introduction}

Levcromakalim is a benzopyran compound BRL38227 which opens up the potassium channel via hyperpolarization of the cell membranes, mediated by the activation of the potassium channels. Levcromakalim, being a potassium channel opener acts by preventing calcium influx and thus could possibly be useful in managing blood pressure and decreasing gastric acid secretion [1]. It has also been shown to act as a bronchodilator [1]. Previous work done using experimental animal models have shown its ability to decrease blood pressure due to its relaxant activity in the vascular smooth muscle [2]. However its role in the management of diabetes mellitus is an area yet to be investigated.

Diabetes mellitus is a disease/group of syndromes characterized by chronic hyperglycaemia as a result of either lack of insulin or resistance to its action; hence there is altered metabolism of lipids, carbohydrates and proteins and increased risk of complications from vascular disease [3].

All forms of Diabetes Mellitus are due to either a decrease in the circulating concentration of insulin (insulin deficiency seen in type 1) or a decrease in the response of peripheral tissues to insulin (insulin resistance seen in type 2). These abnormalities are what lead to alterations in metabolism of carbohydrates, lipids, ketones and amino acids, which are the features of hyperglycemia. In both types of diabetics, glucagon levels which are usually elevated in untreated patients, oppose the effect of insulin on the liver by stimulating glycogenolysis and gluconeogenesis, but it has relatively little effect on peripheral utilization of glucose. Thus in the diabetics with either insulin resistance or deficiency, there is increased hepatic glucose production, decreased peripheral glucose uptake and decreased production of glycogen from glucose in the liver [4].

The prevalence of diabetes mellitus (DM) is increasing at a much faster rate in the developing countries than in the developed nations. There are more than 125 millon persons with Diabetes Mellitus in the world today [3] and it is the leading cause of end-stage kidney disease, cardiomyopathy and heart attacks, strokes, retinal degeneration leading to blindness, and non-traumatic amputations [5].

Considering the high incidence of hypertension and diabetes and the positive impact levcromakalim has on hypertension via reduction of blood pressure, this work is thus geared towards ascertaining if potassium channel opening has any effect on diabetes mellitus by investigating some biochemical parameters.

${ }^{*}$ Corresponding author: $\mathrm{O} \mathrm{J}$ Owolabi, Department of Pharmacology and Toxicology, Faculty of Pharmacy, University of Benin, Benin City, Edo State Nigeria, Tel: +2348034120318; E-mail: josphineomo@yahoo.com, owolabi@ uniben.edu

Received August 18, 2011; Accepted December 14, 2011; Published Decembe 21, 2011

Citation: Owolabi OJ, Omogbai EKI (2011) Levcromakalim (BRL38227) A Potassium Channel Activator Alters Some Biochemical Parameters in Streptozotocin- Induced Diabetic Rats. J Diabetes Metab 2:159. doi:10.4172/21556156.1000159

Copyright: ( 2011 Owolabi OJ, et al. This is an open-access article distributed under the terms of the Creative Commons Attribution License, which permits unrestricted use, distribution, and reproduction in any medium, provided the original author and source are credited. 
Citation: Owolabi OJ, Omogbai EKI (2011) Levcromakalim (BRL38227): A Potassium Channel Activator Alters Some Biochemical Parameters in Streptozotocin- Induced Diabetic Rats. J Diabetes Metab 2:159. doi:10.4172/2155-6156.1000159

Page 2 of 5

\section{Materials and Methods}

\section{Animals}

Albino rats were obtained from the Animal house, Department of Pharmacology and Toxicology, Faculty of Pharmacy, University of Benin and the animal house of Department of Physiology, Ambrose Alli University, Ekpoma, Edo State. Those from Ekpoma, were given two weeks acclimatization time before being subjected to the particular experimental protocol. Animals weighed between 250 and $300 \mathrm{~g}$. They were allowed free access to water or a particular solution and fed on standard diet (Bendel Feeds and Flour Mills Ewu, Edo State). Depending on the group, animals were housed 5 in a cage with a 12 hour light-dark cycle.

Approval for the work was obtained from the Faculty of Pharmacy Ethical Committee on the use of Animals for experiments.

Animals were handled according to the standard protocols for the use of laboratory animals (National Institute of Health, USA: Public Health Service Policy on Humane care and use of Laboratory animals, 2002).

\section{Drugs and chemicals}

Streptozotocin (Sigma-Aldrich ,UK) prepared in a $0.1 \mathrm{M}$ citrate buffer with a PH of 4.5, Glibenclamide (Smith Kline Beecham, UK ), Amlodipine(Dr Reddy's Laboratories, UK), Metformin (Merck Sante) , Insulin (Sigma-Aldrich, UK ), Levcromakalim (Smith Kline Beecham, UK ), Creatinine kit (Randox UK), Urea kit (Randox UK).

\section{Experimental procedure for potassium modulation}

Rats (10 groups of 5 each) received $75 \mu \mathrm{g} / \mathrm{kg} /$ day of levcromakalim intraperitoneally for 4 consecutive weeks [6].

Following treatment with levcromakalim the first five groups received normal saline $(2 \mathrm{ml} / \mathrm{kg})$, glibenclamide $(5 \mathrm{mg} / \mathrm{kg})$, metformin (350 mg/kg), insulin (5 and $10 \mathrm{i} . \mathrm{u} / \mathrm{kg}$ subcutaneously)respectively. All other administrations were done orally via an oro-gastric syringe. In the next 5 groups of 5 each, experimental diabetes mellitus was induced using $60 \mathrm{mg} / \mathrm{kg}$ of streptozotocin in a $0.1 \mathrm{M}$ citrate buffer solution [7]. Diabetes was confirmed 3 days later using the glucose oxidase method.
Only rats with blood glucose levels greater than $200 \mathrm{mg} / \mathrm{dl}$ were used. Having confirmed diabetes, immediately the 5 groups received normal saline, glibenclamide $(5 \mathrm{mg} / \mathrm{kg})$, metformin $(350 \mathrm{mg} / \mathrm{kg})$ [8] and insulin (20 and $40 \mathrm{i} . \mathrm{u} / \mathrm{kg}$ ) respectively [9].

After each drug administration, the rats were kept in metabolic cages separately following adaptation to the metabolic cages. The $24 \mathrm{~h}$ urine was collected and blood samples $(5 \mathrm{ml})$ were withdrawn via cardiac puncture. The levels of plasma and urine creatinine and urea, creatinine clearance, plasma glucose and urine volume were determined 24 hours after drug administration.

\section{Statistical analysis}

Data are presented as the mean \pm standard error of the mean (S.E.M) and $\mathrm{n}$ represents the number of rats per group.

Comparisons were made where appropriate by One-way ANOVA (GraphPad Prism Software, UK) with Tukey post hoc. A value of $\mathrm{p}<$ 0.05 indicates significant differences in all cases.

\section{Results}

Effect of (levcromakalim) on the creatinine and urea of normal and streptozotocin induced diabetic rats

The results on creatinine and urea (plasma and urea) for normal and diabetic rats are presented in (Table 1 and Table 2) respectively.

For the normal rats, the potassium channel activated group (PCA) had a significantly lower plasma creatinine and urine urea levels $(\mathrm{p}<0.05)$, while the urine creatinine was significantly higher $(\mathrm{p}<0.05)$ in comparison with the control group. Treatments of the PCA group with glibenclamide, significantly increased the level of the plasma urea in comparison with the untreated PCA group, with metformin, a significant decrease in the urine urea value was noted, while treatment with insulin $(5 \mathrm{i} . \mathrm{u} / \mathrm{kg})$ significantly increased both the plasma creatinine and urea levels in comparison with the untreated PCA group. All the animals in the PCA group treated with $10 \mathrm{i.u} / \mathrm{kg}$ of insulin died before 24 hours, probably from hypoglycemia.

The result in (Table 2) shows that the potassium channel activated diabetic rats $(P C A D)$ had a significantly lower $(p<0.05, p<0.0001)$

\begin{tabular}{|c|c|c|c|c|}
\hline \multirow[b]{2}{*}{ Treatment } & \multicolumn{2}{|c|}{ Creatinine $(\mathrm{mmol} / \mathrm{l})$} & \multicolumn{2}{|c|}{ Urea (mmol/l) } \\
\hline & plasma & urine & plasma & urine \\
\hline $\mathrm{C}(2 \mathrm{ml} / \mathrm{kg})$ & $0.21 \pm 0.03$ & $2.67 \pm 0.62$ & $9.27 \pm 0.37$ & $785.85 \pm 116.90$ \\
\hline Lev & $0.12 \pm 0.02^{b}$ & $9.57 \pm 2.29^{b}$ & $8.64 \pm 1.28$ & $128.96 \pm 6.10^{\mathrm{b}}$ \\
\hline LevG & $0.18 \pm 0.04$ & $8.66 \pm 0.72$ & $19.65 \pm 3.71^{\mathrm{ab}}$ & $125.06 \pm 10.37$ \\
\hline LevM & $0.14 \pm 0.03$ & $10.39 \pm 1.42$ & $9.85 \pm 1.72$ & $47.0 \pm 6.40^{\mathrm{ac}}$ \\
\hline Levl5 & $0.20 \pm 0.02^{\mathrm{ab}}$ & $10.99 \pm 1.29$ & $18.89 \pm 1.29^{a b}$ & $38.37 \pm 5.32^{\mathrm{ac}}$ \\
\hline Levl10* & - & - & - & - \\
\hline
\end{tabular}

Values are mean \pm SEM

$(\mathrm{n}=5$ per group)

${ }^{b} p<0.05$ significantly different from the control and ${ }^{a b} p<0.05$, ${ }^{a c} p<0.0001$ significantly different from the group treated with levcromakalim

* all died

C: Control (normal saline group)

Lev : Potassium channel activated group

LevG: Potassium channel activated group treated with glibenclamide

LevM : Potassium channel activated group treated with metformin

Levl5 : Potassium channel activated group treated with $5 \mathrm{i} . \mathrm{u} / \mathrm{kg}$ insulin

Levl10: Potassium channel activated group treated with $10 \mathrm{i} . \mathrm{u} / \mathrm{kg}$ insulin

Table 1: The effects of levcromakalim on the creatinine and urea of normal Wistar rats treated with glibenclamide $(5 \mathrm{mg} / \mathrm{kg}), \mathrm{metformin}(350 \mathrm{mg} / \mathrm{kg})$ and insulin $(5 \& 10$ i.u/kg). 
Citation: Owolabi OJ, Omogbai EKI (2011) Levcromakalim (BRL38227): A Potassium Channel Activator Alters Some Biochemical Parameters in Streptozotocin- Induced Diabetic Rats. J Diabetes Metab 2:159. doi:10.4172/2155-6156.1000159

Page 3 of 5

\begin{tabular}{|l|l|l|l|l|}
\hline & \multicolumn{2}{|c|}{ Creatinine (mmol/l) } & \multicolumn{2}{l}{ Urea (mmol/l) } \\
\hline Treatment & Plasma & urine & plasma \\
\hline D & $0.26 \pm 0.03$ & $2.64 \pm 0.46$ & $11.23 \pm 0.49$ \\
\hline LevD & $0.22 \pm 0.03$ & $8.09 \pm 1.12^{\mathrm{b}}$ & $16.63 \pm 2.79$ & $473.33 \pm 81.5$ \\
\hline LevDG & $0.21 \pm 0.02$ & $10.23 \pm 1.88$ & $18.89 \pm 1.30$ & $46.88 \pm 12.2^{\mathrm{a}}$ \\
\hline LevDM & $0.19 \pm 0.04$ & $12.11 \pm 1.46$ & $18.46 \pm 1.28$ & $16.54 \pm 1.91$ \\
\hline LevDI20 & $0.18 \pm 0.02$ & $8.32 \pm 2.04$ & $45.64 \pm 5.03$ & $17.90 \pm 1.88$ \\
\hline LevDI40 & $0.26 \pm 0.02$ & $10.53 \pm 3.06$ & $27.68 \pm 4.26$ & $28.26 \pm 6.89$ \\
\hline
\end{tabular}

Values are mean \pm SEM.

( $\mathrm{n}=5$ per group)

${ }^{a} p<0.0001,{ }^{b} p<0.05$ significantly different from the diabetic group .

D: Diabetic group

LevD: Potassium channel activated diabetic group

LevDG: Potassium channel activated diabetic group treated with glibenclamide

LevDM: Potassium channel activated diabetic group treated with metformin

LevDI20: Potassium channel activated diabetic group treated with $20 \mathrm{i} . \mathrm{u} / \mathrm{kg}$ insulin

LevDI40 : Potassium channel activated diabetic group treated with $40 \mathrm{i} . \mathrm{u} / \mathrm{kg}$ insulin

Table 2: The effects of levcromakalim on the creatinine and urea of streptozotocin-induced diabetic rats treated with glibenclamide $(5 \mathrm{mg} / \mathrm{kg})$, $\mathrm{metformin}(350 \mathrm{mg} / \mathrm{kg})$ and insulin (5 \& $10 \mathrm{i} . \mathrm{u} / \mathrm{kg})$.

urine creatinine and urea levels respectively in comparison with the untreated diabetic rats. It was however noted that treatment with glibenclamide, metformin or insulin produced no significant changes in both the creatinine and urea levels (plasma and urine) of the PCAD group.

Effect of (levcromakalim) on the plasma glucose and creatinine clearance of normal and streptozotocin induced diabetic rats

The results on the creatinine clearance for both normal and diabetic rats are presented in (Figures 1 and 2) respectively. The creatinine clearance of the PCA group was significantly higher $(\mathrm{p}<0.05$, Figure 1$)$ than that of the control group. This raised creatinine clearance value

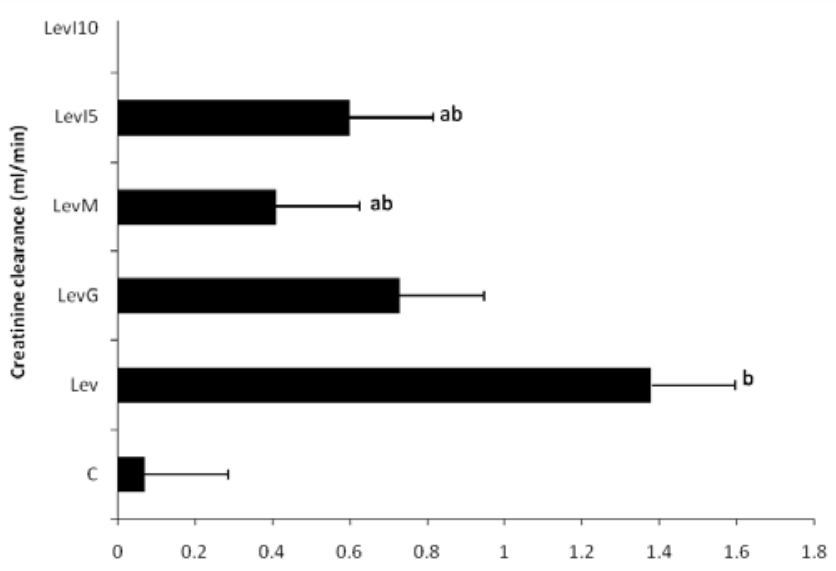

Values are mean \pm SEM

( $\mathrm{n}=5$ per group).

${ }^{\mathrm{b}} \mathrm{P}<0.05$ significantly different from the control, ${ }^{\mathrm{ab}} \mathrm{P}<0.05$ significantly different from the group treated with only levcromakalim.

C: Control (normal saline group)

Lev: potassium channel activated group

LevG: potassium channel activated group treated with glibenclamide

LevM: potassium channel activated group treated with metformin

Levl: potassium channel activated group treated with insulin

Levl10 : all died

Figure 1: The effect of levcromakalim treatment on the creatinine clearance of normal Wistar albino rats treated with glibenclamide, metformin and insulin.

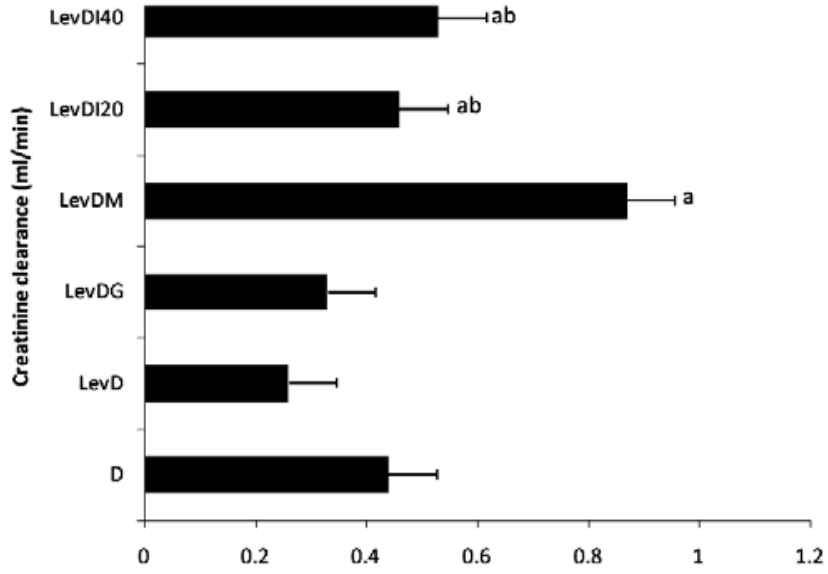

Values are mean \pm SEM.

( $n=5$ per group).

${ }^{\mathrm{a}} \mathrm{P}<0.0001$ and ${ }^{\mathrm{ab}} \mathrm{P}<0.05$ significantly higher from the diabetic group treated with levcromakalim.

D: diabetic group

LevD : potassium channel activated diabetic group

LevDG : potassium channel activated diabetic group treated with glibenclamide LevDM: potassium channel activated diabetic group treated with metformin LevDI20/40 : potassium channel activated diabetic group treated with insulin

Figure 2: The effect of levcromakalim treatment on the creatinine clearance of streptozotocin-induced rats treated with glibenclamide, metformin and insulin.

for the PCA group was however significantly decreased $(\mathrm{p}<0.05)$ on treatment with metformin and insulin (5 i.u/kg).

For the diabetic rats, interestingly, no significant difference was observed in the creatinine clearance of the untreated diabetic and PCAD groups (Figure 2). Treatment however of the PCAD group separately with metformin and insulin (20 and $40 \mathrm{i} . \mathrm{u} / \mathrm{kg}$ ) produced a significant increase in the creatinine clearance $(\mathrm{p}<0.0001, \mathrm{p}<0.05)$ respectively in comparison with the untreated PCAD group.

The results on plasma glucose of STZ-induced diabetic rats are shown in (Table 3). The plasma glucose of the diabetic rats were significantly higher than control $(\mathrm{p}<0.05)$. Though treatment with levcromakalim in the diabetic rats produced a slight increase in their 
Citation: Owolabi OJ, Omogbai EKI (2011) Levcromakalim (BRL38227): A Potassium Channel Activator Alters Some Biochemical Parameters in Streptozotocin- Induced Diabetic Rats. J Diabetes Metab 2:159. doi:10.4172/2155-6156.1000159

plasma glucose levels, this was however not significant. Treatment with metformin and glibenclamide seems to worsen the hyperglycemia of the diabetic rats in the presence of levcromakalim. Here a significant increase was observed $(\mathrm{p}<0.05)$. Reduction of hyperglycemia was only possible on treatment with insulin $(40 \mathrm{i} . \mathrm{u} / \mathrm{kg})$. Here a significant reduction was noted $(\mathrm{p}<0.05)$.

\section{Effect of (levcromakalim) on the 24 hour urine volume of normal and streptozotocin induced diabetic rats}

Figures 3 and 4 presents result on the volume of urine of normal and diabetic rats respectively. For the normal rats (Figure 3), a significantly

\begin{tabular}{|c|c|}
\hline Treatment & Plasma glucose $(\mathrm{mg} / \mathrm{dl})$ \\
\hline C & $83.46 \pm 7.95^{\mathrm{ab}}$ \\
\hline D & $223.49 \pm 2.93$ \\
\hline LevD & $240.0 \pm 2.02$ \\
\hline LevDG & $274.3 \pm 19.96^{\mathrm{a}}$ \\
\hline LevDM & $321.6 \pm 8.09^{\mathrm{a}}$ \\
\hline LevDI20 & $257.0 \pm 32.9$ \\
\hline LevDI40 & $194.4 \pm 5.17^{\mathrm{b}}$ \\
\hline
\end{tabular}

Values are mean \pm SEM

( $n=5$ per group).

${ }^{a b} p<0.0001$ and ${ }^{a} p<0.05$ significantly higher than the diabetic group and ${ }^{b} p<0.05$ significantly lower than the diabetic group.

C: Control group

D: Diabetic group

LevD: Potassium channel activated diabetic group

LevDG: Potassium channel activated diabetic group treated with glibenclamide LevDM: Potassium channel activated diabetic group treated with metformin LevDI20: Potassium channel activated diabetic group treated with $20 \mathrm{i} . \mathrm{u} / \mathrm{kg}$ insulin

LevDI40 : Potassium channel activated diabetic group treated with $40 \mathrm{i} . \mathrm{u} / \mathrm{kg}$ insulin

Table 3: The effects of levcromakalim on the plasma glucose of streptozotocininduced diabetic rats treated with glibenclamide $(5 \mathrm{mg} / \mathrm{kg})$, metformin (350 mg/ $\mathrm{kg}$ ) and insulin (5 \& $10 \mathrm{i} . \mathrm{u} / \mathrm{kg})$.

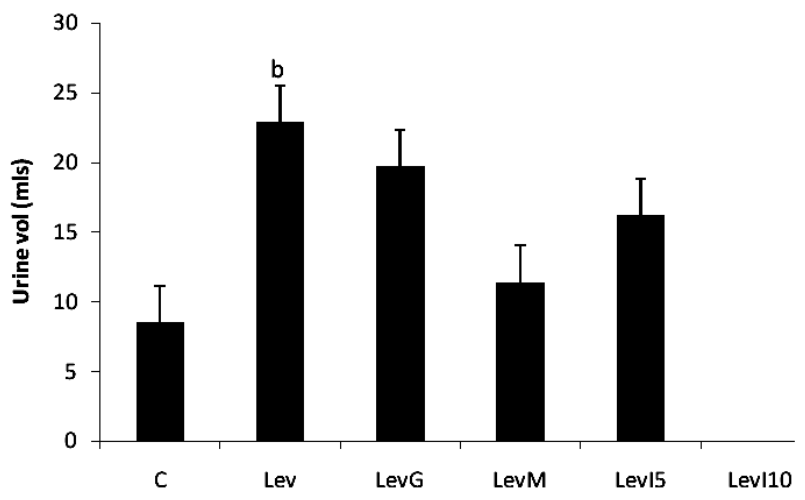

Values are mean + SEM

$(n=5$ per group).

${ }^{\mathrm{b}} \mathrm{P}<0.05$ significantly different from the control.

C: Control (normal saline group)

Lev: potassium channel activated group

LevG: potassium channel activated group treated with glibenclamide

LevM: potassium channel activated group treated with metformin

Levl: potassium channel activated group treated with insulin

Levl10 : all died

Figure 3: The effect of levcromakalim treatment the on urine output of normal Wistar albino rats treated with glibenclamide, metformin and insulin.

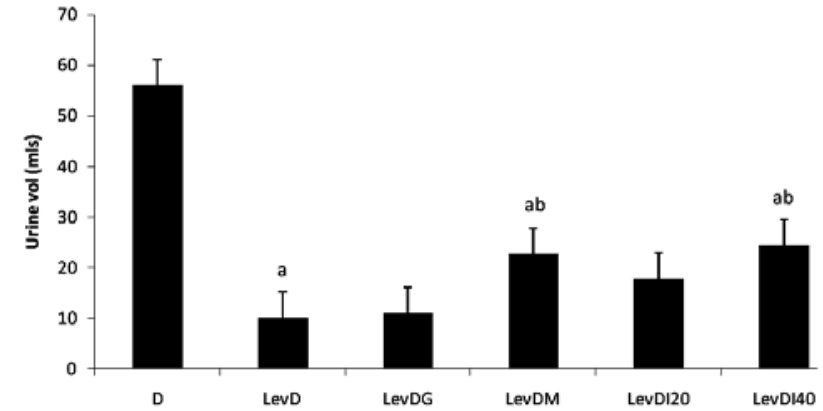

Values are mean \pm SEM

( $n=5$ per group).

a $\mathrm{P}<0.0001$ significantly different from the diabetic group and ${ }^{\mathrm{ab}} \mathrm{P}<0.05$ significantly different from the diabetic group treated with levcromakalim .

D: diabetic group

LevD : potassium channel activated diabetic group

LevDG : potassium channel activated diabetic group treated with glibenclamide LevDM: potassium channel activated diabetic group treated with metformin LevDI20/40 : potassium channel activated diabetic group treated with insulin

Figure 4: The effect of levcromakalim treatment on the urine output of streptozotocin- induced diabetic rats treated with glibenclamide, metformin and insulin.

higher $(\mathrm{p}<0.05)$ urine volume was observed for the PCA group in comparison with the control group. Treatment with glibenclamide, metformin or insulin separately produced no significant change in the urine volumes of the PCA group.

Diabetic rats had a significantly higher urine volume in comparison with the untreated PCAD group $(\mathrm{p}<0.0001$, Figure 4$)$. The PCAD groups treated with metformin and insulin (40 i.u/kg) separately had a significantly higher $(\mathrm{p}<0.05)$ urine volume in comparison with those not treated.

\section{Discussion}

The 24 hour urine volume of normal rats chronically treated with levcromakalim was significantly higher than that of rats not exposed to levcromakalim. Treatment of the PCA group with glibenclamide, metformin or insulin produced no significant difference in the volume of urine in comparison with those untreated. In the diabetic rats where polyuria is a problem, a significant decrease is seen on treatment with levcromakalim, i.e the 24 hour urine volume of the PCAD group was significantly lower than the untreated diabetic rats. An increase was observed in the 24 hour urine volume in the PCAD rats treated with metformin and insulin. Though there was an increase on treatment with these drugs, it was however still far lower than the urine output of the untreated diabetic rats $(\mathrm{p}<0.05)$. It is also interesting to observe that treatment with levcromakalim had no significant effect on the plasma glucose of the diabetic rats. Levcromakalim combined with insulin may thus be a good option for the diabetic individuals with co-existing hypertension considering both its blood pressure lowering property and its significant lowering effect on the 24 hour urine volume of the diabetic rats.

Treatment of normal rats with levcromakalim significantly decreased the plasma creatinine, urine urea and increased the urine creatinine levels. For diabetic rats, a similar effect was noted on treatment with levcromakalim. The urine creatinine was significantly increased while the urine urea was decreased. The significant increase in urine creatinine for both normal and diabetic rats and the significant decrease in plasma creatinine in normal rats is a strong indication 
Citation: Owolabi OJ, Omogbai EKI (2011) Levcromakalim (BRL38227): A Potassium Channel Activator Alters Some Biochemical Parameters in Streptozotocin- Induced Diabetic Rats. J Diabetes Metab 2:159. doi:10.4172/2155-6156.1000159

of the positive impact treatment with levcromakalim may have on the glomerular filtration rate. Creatinine levels in blood and urine are usually used to calculate the creatinine clearance which reflects the glomerular filtration rate. The glomerular filtration rate (GFR) is important clinically because it is a measure of the renal function. High creatinine level in the urine indicates the ideal, while a high blood or plasma creatinine level may indicate malfunctioning of the kidneys. GFR is so important in assessing the excretory function of the kidneys [10]. Treatment of the PCA group with insulin produced a significant increase in plasma creatinine, urea and the urine urea levels. This increase in the plasma creatinine was however not different from the values of the normal rats. The increased plasma level of the urea and decreased level of urea in the urine produced on treatment with insulin may be a disadvantage as plasma concentration of waste products like urea is usually used to determine renal function [11]. In the PCAD rats, treatment with glibenclamide, metformin or insulin produced no significant difference in the plasma and urine creatinine and urea levels in comparison with those not treated with the different drugs.

Chronic treatment with levcromakalim significantly increased the creatinine clearance in normal rats. The level of creatinine excreted by urine was increased on chronic treatment with levcromakalim. This could mean that treatment with levcromakalim enhances the excretory function of the kidney this is further seen in the raised creatinine clearance level which is a measure of the glomerular filtration rate of the kidney. However for the diabetic rats, treatment with levcromakalim had no significant effect on the creatinine clearance. Levcromakalim has been reported using experimental animal models to decrease blood pressure due to its relaxant activity in the vascular smooth muscle [2], hence its reno-protective effect observed may be linked to its blood pressure lowering abilities.

Treatment of the PCA group with metformin and insulin produced a significant decrease in the creatinine clearance in comparison with those untreated. However the opposite (significant increase in creatinine clearance) was noticed on treatment of the PCAD group with metformin and insulin. So for the diabetic individuals on levcromakalim, treatment with insulin or metformin is encouraged as treatment with either drug increases the creatinine clearance and hence the GFR

\section{Conclusion}

The present study was to determine the effects of levcromakalim on the creatinine and urea, urine volume and creatinine clearance of diabetic rats.

Levcromakalim decreased the volume of 24 hour urine in diabetic rats, the plasma creatinine and increased the urine creatinine in both normal and diabetic rats, while an increase in the creatinine clearance was observed only in normal rats (Reno-protective effect).

This study points to a protective effect on renal function probably linked to its hypotensive effect. Further work (chronic effect) is on to ascertain its exact mechanism of action.

\section{References}

1. Hamilton TC, Beerahee A, Moen JS, Price RK, Ramji JV, et al. (1986) Cardiovascular effects of levcromakalim. Cardiovascular Drug Reviews 11: 199-122.

2. Buckingham RE (1988) Studies on the anti-vasoconstrictor activity of BRL34915 in spontaneously hypertensive rats, a comparison with nifedipine. Br J Pharmacol 93: 541-552.
3. Davis SN and Granner DK (2001). Insulin oral agents and the pharmacology of the endocrine pancreas. In: Hardman JG and Limbird LE. Goodman and Gliman's, Pharmacological basis of Therapeutics. 10th edition, pp: 115-154

4. Katzung BG (2001) Basic and Clinical Pharmacology, 8th edition. McGraw Hill Publishers p.725.

5. Engelgau MM, Geiss LS, Saadine JB, Boyle JP, Benjamin SM, et al. (2004) The evolving diabetes burden in the United States. Ann intern med 140: 945-950.

6. Trongvanichnam K, Mitsui-Saito M, Ozaki H, Karaki H (1996) Effects of chronic oral administration of levcromakalim on invitro contractile responses of arterial smooth muscle. Eur J Pharmacol 303: 39-45.

7. Frode TS, Medeiros YS (2008) Animal models to test drugs with potential antidiabetic activity. J Ethnopharmacol 115: 173-183.

8. Stumvoll M, Nurjaham N, Perriello G, Dailey G, Gerich JE (1995) Metabolic effects of metformin in Non-insulin dependent Diabetes mellitus. N Engl J Med 333: $550-554$

9. Jafari MR, Zarrindast MR, Djahanguiri B (2004) Effects of different doses of glucose and insulin on morphine state dependent memory of passive avoidance in mice. Psychopharmacology (Berl) 175: 457-462.

10. Gross JL, De Azevedo MJ, Silveiro SP, Canani LH, Caramori ML, et al. (2005) Diabetic nephropathy: diagnosis, prevention, and treatment. Diabetes Care 28 164-176.

11. Bazari H, Jaff MR, Mannstadt M, Yan S (2007) Case records of a Massachusetts General Hospital. Case 7-2007. A 59-year old woman with diabetic renal disease and non healing ski ulcers. N Engl J Med 358: 827-838. 